\title{
Hunting down giant cells in deep stellar convective zones
}

\author{
Nicolas Bessolaz and Allan Sacha Brun \\ CEA Saclay, DSM/IRFU/SAp, 91191 Gif-sur-Yvette Cedex, France \\ email: Nicolas.Bessolaz@cea.fr
}

\begin{abstract}
D high resolution simulations for the convective zone of a $4 \mathrm{Myr}$ old $0.7 M_{\odot}$ pre-main sequence star in gravitational contraction are carried out with different radial density contrast using the pseudo spectral ASH code (Brun et al. 2004). We extract giant cells signal from the complex surface convective patterns by using a wavelet analysis. We then characterize them by estimating their lifetime and rotation rate according to the density contrast.
\end{abstract}

Keywords. convection - stars : pre-main sequence, low-mass, rotation - turbulence

\section{Introduction}

Understanding the dynamical properties of deep convective zones is of fundamental interest to explain the evolution and redistribution of heat, energy and angular momentum transport in pre-main sequence stars. Detection of giant cells already in our Sun is very difficult (LaBonte et al. 1981, Lisle et al. 2004) since they are merged within stronger signals like granulation or supergranulation which have to be removed correctly. They further demand to substract the global differential rotation of the Sun, the limb effect and disentangle possible line shifts due to the solar magnetic field in active regions. Full sphere simulations resolving only the largest scales of turbulent convection down to supergranulation with mean flows, combined with a specific wavelet analysis (Starck et al. 2006) are suitable tools to probe these giant cells and study their properties.

\section{Convective structures and giant cells properties}

Four different models with an e-folding increasing radial density contrast from 13 to 272 are computed with a common outer edge at $R=0.98 R_{*}$ and a fixed surface density scale height $H_{\rho}=10 \mathrm{Mm}$. The initial stellar state is obtained from the CESAM code (Morel 1997). Typical run for these simulations lasts 3000 days, i.e. 10 convective overturning time and the rms Reynolds number in the mid layer varies between 100 and 700 .

The different aspect ratio $R_{*} / \Delta R$ between models (from 14 to 2.2 with the increasing density contrast) has in appearance little influence on the convective patterns close to the stellar surface as shown in Figure 1 for the radial velocity. Thus, provided that the level of turbulence is sufficient, the small-scale convection patterns are really linked to the local density scale height which is common to all models and equal to $10 \mathrm{Mm}$. Hence the difficulty to extract information about the underlying giant cells from the surface. The 3D structure of the convective patterns are similar whatever the latitude is for the $\Delta \rho=13,37$ models whereas the $\Delta \rho=100,272$ models show convective cells extended through the whole convective zone and parallel to the rotation axis at high latitude i.e. convective structures modify their orientation to accomodate for the Coriolis force in these models where the Rossby number is below unity.

After having detected a large scale signal with our wavelet analysis (see Bessolaz \& Brun 2011 for details), a time correlation analysis similar to the work of Miesch et al. 

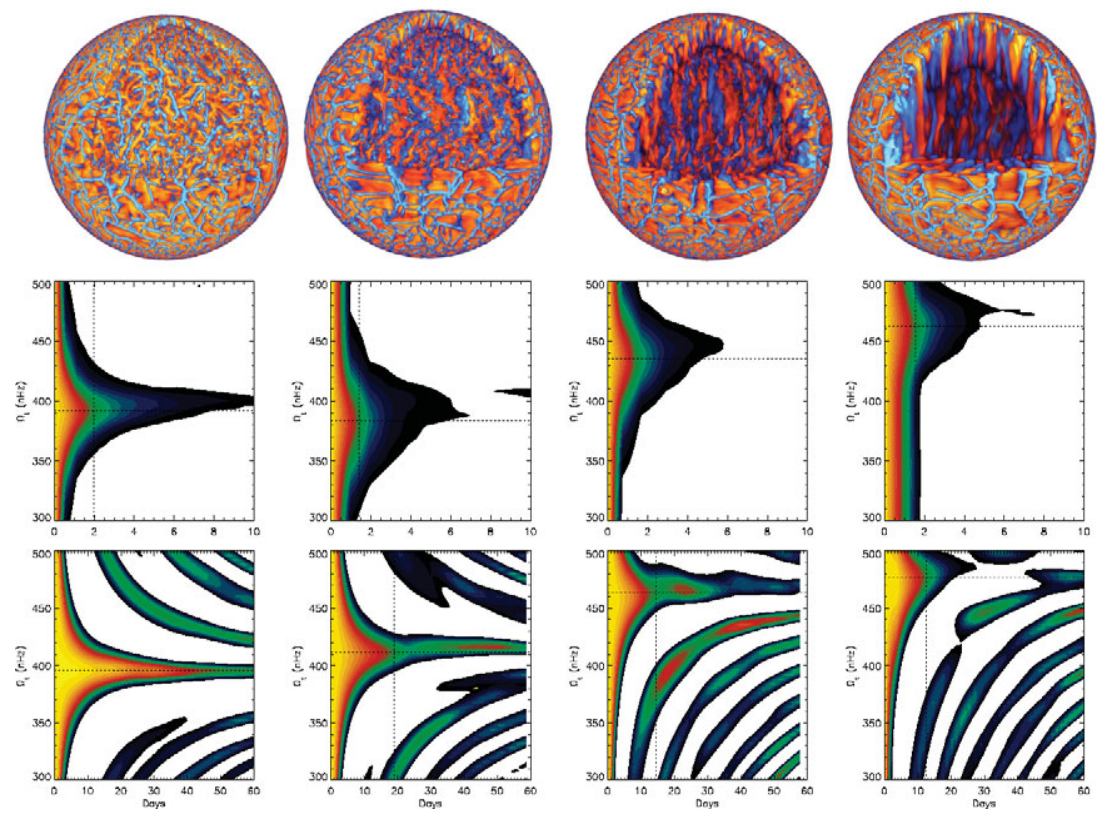

Figure 1. Top : 3D rendering (done with SDVision, Pomarede et al. 2008) of the radial velocity pattern cropped in one quadrant in the North hemisphere to highlight the convective structures in depth for the different models (by increasing the density contrast from left to right).

Bottom : Autocorrelation function computed close to the surface $\left(R=0.97 R_{*}\right)$ and for the latitudinal band $\left[0-20^{\circ}\right]$ according to the tracking rate for all models both for the full image (upper figures) and only the largest scale (lower figures). Notice the different much larger temporal range for the large scale analysis.

(2008) is performed both on the full convective flow and on the reconstructed largest spatial scales of the wavelet analysis. This helps to quantify the lifetime of these large scale structures detected over two stellar periods for each model (see Fig. 1). The optimal tracking velocity $\Omega_{o p t}$ which maximize the autocorrelation function (acf) defines the lifetime of giant cells for a threshold of 0.5 of the acf function. For the full images including a large range of scales, the lifetime is really similar for all models around 1.52 days whereas large scale structures have lifetimes greater than 10 days. The lifetime of these large scale structures seems also to decrease with the density contrast from $\tau=60$ days till $\tau=12$ days because they undergo much distortions due to the stronger differential rotation in these high density contrast models. Giant cells are elongated in the North/South direction with a varying latitudinal extension linked to the depth of the convection zone. Finally, they have a proper tracking rate really greater than the local differential rotation and the discrepancy increases with the density contrast.

Acknowledgements : We acknowledge funding by ERC through grant STARS2 \#207430.

\section{References}

Bessolaz, N. \& Brun, A. S. 2011, ApJ, 728, 115

Brun, A. S., Miesch, M. S., \& Toomre, J. 2004, ApJ, 614, 1073

Labonte, B. J., Howard, R., \& Gilman, P. A. 1981, ApJ, 250, 796

Lisle, J. P., Rast, M. P., \& Toomre, J. 2004, ApJ, 608, 1167

Miesch, M. S., Brun, A. S., DeRosa, M. L., \& Toomre, J. 2008, ApJ, 673, 557

Morel, P. 1997, A\&AS, 124, 597

Pomarede et al. 2008, Astronomical Society of the Pacific Conference Series, vol. 386, 327

Starck, J.-L., Moudden, Y., Abrial, P., \& Nguyen, M. 2006, A\&\&A, 446, 1191 